\title{
La transmission et l'articulation des savoirs dans une communauté de pêche au Québec
}

Maryvonne Merri,

Département de psychologie- Université du Québec à Montréal, Canada, merri.maryvonne@uqam.ca 


\section{REVUE HYBRIDE DE L’ÉDUCATION}

\section{Résumé}

Cet article met en regard La transmission des savoirs de Delbos et Jorion avec les récits de formation de pêcheurs québécois. Les équipages québécois ne sont plus uniquement familiaux et les pêcheurs s'inscrivent autant dans une identité biographique pour soi que dans une identité communautaire. Au sein d'une solidarité organique entre le capitaine et les hommes de pont, les formes d'apprentissage, toutes valorisées, varient selon les tâches : les pêcheurs différencient les savoirs locaux implicites, appartenant au capitaine, des apprentissages par l'observation d'autrui, par l'école ou avec des experts. L'innovation technologique est une nouvelle occasion d'activité constructive selon ces pêcheurs.

Mots-clés : Delbos et Jorion ; pêche ; Québec ; transmission des savoirs ; solidarité organique 


\section{8}

REVUE HYBRIDE DE L'ÉDUCATION

\section{Problématique}

L'activité des pêcheurs, comme celle de tout professionnel agissant avec et sur la nature, prend part à un écosystème naturel et à un écosystème politique, économique et culturel. Les actions du pêcheur, en étant soumises à cette double complexité naturelle et humaine, sont incertaines (Mayen, 2016). En concevant et en adoptant des instruments et des systèmes instrumentaux tant matériels (engins de pêche) que règlementaires (brevets de pêche, normes de sécurité), symboliques (valeurs de solidarité) ou cognitifs (savoirs et techniques), les pêcheurs développent leur capacité de prévoyance et de prévision (Bruner, 1996 ; Del Corso, 2008).

À partir du XVIIlème siècle, les encyclopédistes décrivent les techniques des pêcheurs (Duhamel du Monceau, 1776 ; Merri et Doualot, 2013). Ils annoncent l'arrivée des scientifiques, de la formation maritime (Biget, 2005, 2009), du conseil technique (Boudreau et Myre, 1998; Deschamps, 2009), des législateurs et des acteurs économiques. Une solidarité organique (Durkheim, 1893) fondée sur une articulation des fonctions (Merri et al., 2015) se substitue partiellement à une solidarité mécanique (Durkheim, 1893) familiale fondée sur une communauté de valeurs et de destin.

Cette nouvelle solidarité constitue possiblement une opportunité pour les pêcheurs, selon qu'ils parviennent à coordonner leurs actions sur le bateau, à articuler des savoirs et des technologies d'origines variées, qu'ils acquièrent des compétences discursives et stratégiques face aux autres acteurs (Lorino, 2013; Merri et Képhaliacos, 2016) et qu'ils réexaminent leurs raisons d'agir (Merri et Képhaliacos, 2016).

Dans cet article, nous explorerons les témoignages de pêcheurs appartenant à une même communauté de pêche du Québec. Quels sont les savoirs mobilisés par la pêche ? Comment leur ont-ils été transmis et comment les transmettent-ils ? Quels sont les savoirs récemment introduits dans l'activité de pêche et les articulent-ils avec les savoirs antérieurs?

Dans une première partie, nous dégagerons les principales thèses de La transmission des savoirs, ouvrage de Delbos et Jorion publié en 1984 sur les métiers maritimes et, à ce jour, unique pour ce secteur d'activité. Dans une seconde partie, nous mettrons ces thèses en regard avec les discours actuels des pêcheurs de la communauté de pêche québécoise étudiée. 


\section{$<$}

REVUE HYBRIDE DE L'ÉDUCATION

\section{Cadre conceptuel}

L'ouvrage d'anthropologie de l'éducation de Delbos et Jorion (1984) ${ }^{1}$ porte sur les métiers maritimes (récolte du sel dans les marais salants, ostréiculture et petite pêche) de l'Ouest de la France en s'appuyant, en particulier, sur des récits de formation. Cet ouvrage permet de dégager cinq propositions sur la transmission des savoirs pour la pêche qui peuvent être rapprochées des travaux engagés à cette même époque sur la participation dans les communautés de pratique (Lave et Wenger, 1991; Wenger, 2005).

Les trois premières propositions décrivent la pêche comme une communauté de pratique dans laquelle l'apprentissage a lieu par diffusion des savoirs dans la famille (pour un autre exemple, voir les sages-femmes en Amérique Centrale dans Lave et Wenger [1991]). Les quatrième et cinquième propositions sont, par contre, générales à toutes les communautés de pratique (Lave et Wenger, 1991). L'amour-propre et la légitimité de l'apprenti créent la possibilité d'apprendre et d'innover. En effet, la communauté de pratique doit rester orientée vers son futur (Wenger, 2005). Ainsi, dans la pêche, l'introduction de nouveaux engins renouvelle à la fois les pratiques, l'articulation des savoirs et les relations dans la communauté entre les pêcheurs-précurseurs et les autres pêcheurs.

Les enfants ne sont pas ségrégés de l'activité professionnelle des adultes.

Le métier s'apprend non seulement dans le travail, mais également au hasard d'une conversation, d'une réparation d'un engin de pêche à terre par l'observation, l'écoute, les sensations et les émotions :

On apprend le métier par tous les bouts et à tous les détours, parce qu'il est la vie (...). De tout l'entourage sourd cependant un savoir qui peut être reçu : savoirfaire, savoir-dire, savoir-vivre, sont inculqués pêlemêle, découverts par soi-même, mais corrigés si nécessaire. (Delbos et Jorion, 1990, p. 97)

\section{Le savoir professionnel est également un patrimoine symbolique} grâce à la transmission progressive des places au travail.

L'espace du bateau est divisé entre le bas, le pont et le haut. Ce sont autant de places que le novice peut espérer occuper dans sa carrière (Rostaing, 2012). Le jeune n'a d'abord le choix qu'entre quelques

\footnotetext{
${ }^{1}$ Nous utiliserons dans la suite la seconde édition de cet ouvrage, disponible en ligne, permettant ainsi sa lecture intégrale: https://books.openedition.org/editionsmsh/13647?lang=fr
} 


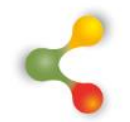

REVUE HYBRIDE DE L'ÉDUCATION

possibilités : « embarquer avec son père, ou avec les frères aînés, si celuici a déjà pris sa retraite, ou rejoindre peut-être l'équipage de celui qui apparaît déjà comme le futur beau-père » (Delbos et Jorion, 1990, p. 26). Lorsqu'il arrive sur le bateau, le novice trouve toujours du travail à faire et il s'agit de faire gagner du temps aux autres membres de l'équipage.

Ainsi, le jeune s'ajuste progressivement aux autres pêcheurs : il accomplit d'abord des tâches pour l'avancement du travail, puis il devient une main-d'œuvre supplémentaire qui libère un marin expérimenté pour des tâches plus spécialisées jusqu'à être une main-d'œuvre complémentaire.

En parvenant à ajuster son activité avec celle des autres membres de l'équipage, le jeune marin devient légitime à participer (Lave et Wenger, 1991) dans la mesure du risque de ses actions. II est alors confronté à l'interdit que Delbos et Jorion (1990) désignent comme le «non du père ».

Le métier finit par "se savoir » sans transmission du savoir par les aînés.

Pour les professionnels rencontrés par Delbos et Jorion (1990), le métier ne s'apprend pas au sens d'«apprendre à l'école». Le jeune réactualise dans la pratique la «confrontation humaine avec la nature » en étant sanctionné par celle-ci et par les aînés. Quatre formes d'apprentissage sont distinguées dans les témoignages (Delbos et Jorion, 1990, p. 4 -5) (tableau 1).

Tableau 1 : Les formes d'apprentissage déclarées par les professionnels de Delbos et Jorion (1990)

\begin{tabular}{|l|l|}
\hline $\begin{array}{l}\text { Le non- } \\
\text { apprentissage }\end{array}$ & "Y'a rien à dire, ça s'apprend pas ce boulot-là.» \\
\hline $\begin{array}{l}\text { L'apprentissage } \\
\text { par frayage }\end{array}$ & $\begin{array}{l}\text { "Tu vis là-dedans, alors t'as pas à réfléchir, ça } \\
\text { se fait tout seul, comme quand tu commences à } \\
\text { marcher... » }\end{array}$ \\
\hline $\begin{array}{l}\text { L'apprentissage } \\
\text { comme mémoire } \\
\text { du « non du père » }\end{array}$ & $\begin{array}{l}\text { "Tu sais pas la connerie que t'as fait, mais au } \\
\text { moins tu sais que t'as fait une connerie... alors } \\
\text { toutes les conneries à ne pas faire tu les } \\
\text { connais. » }\end{array}$ \\
\hline $\begin{array}{l}\text { Une expérience } \\
\text { personnelle et } \\
\text { privée comme } \\
\text { réactualisation de } \\
\text { la confrontation } \\
\text { avec la nature }\end{array}$ & $\begin{array}{l}\text { "Les trucs du métier, personne te les dira. Sauf } \\
\text { si t'as travaillé avec ton père... et encore ! » } \\
\text { "Ça marche ou ça marche pas, si ça marche, tu } \\
\text { continues sans trop te poser de questions... » }\end{array}$ \\
\hline
\end{tabular}




\section{8}

REVUE HYBRIDE DE L'ÉDUCATION

La quatrième forme (tableau 1) concerne l'« intelligence pratique du monde naturel » dans un espace situé, c'est-à-dire des savoirs locaux (Archambault, 1997 ; Geertz, 1986). Le pêcheur doit répondre à la question " que faire ici et maintenant ? » avec ce courant, cette température, ce type de fond. Les seuls moments de transmission de ce savoir sont associés au dévoilement privilégié de secrets professionnels par un ancien :

Souvent, il s'agit de secrets de polichinelle, mais cela n'est pas très important, car il ne s'agit pas vraiment de contenus. Ce qui se transmet là, c'est une bénédiction. (Delbos et Jorion, 1990, p. 184)

Enfin, la rencontre entre l'école et la pratique est source de malentendus entre les générations et les aînés répondent aux «pourquoi » du jeune : «Parce que c'est comme ça !... » (Delbos et Jorion, 1990, p. 8)

\section{Le travailleur développe un amour-propre et une activité propre.}

C'est en étant confronté à la nature et rappelé à l'ordre que le novice finit par savoir ce qui n'est pas transmis explicitement: "Les contenus, dans la mesure où on ne les lui dit pas, le fils va devoir les trouver. Mais qu'est-ce qui le pousse à chercher ? L'“amour-propre" dit-on ici. » (Delbos et Jorion, 1990, p. 89), c'est-à-dire :

(...) le contentement de soi (qui) repose en grande partie sur l'opinion réelle ou imaginaire des autres : il est la partie de l'estime de soi-même qui se construit en fonction de ce que chacun ressent de ce que les autres pensent en vérité ou en imagination de soi. (Mauroy, 2014, p. 74)

À la pêche, plus le professionnel a (du savoir), plus il est (un pêcheur) (Ulmann, 2009) et plus il est reconnu par son père et dans sa communauté.

\section{L'innovation technologique fait évoluer les savoirs de la pratique et est source de différenciation sociale.}

Les professionnels sont à l'affût des innovations technologiques, mais cet investissement est risqué. En effet, il est possible que l'engin n'ait pas une incidence significative sur la rentabilité dans les conditions locales de pêche, cette rentabilité pouvant également s'atténuer en cas de surpêche ou d'innovation. Le nouvel engin requiert également une prise de risque économique et de nouveaux savoirs pour son usage optimal. Enfin, les innovations technologiques mettent en contact les pêcheurs avec de nouveaux acteurs (les techniciens de pêche, les organismes financiers et subventionnaires, les législateurs) au risque de leur autonomie professionnelle. 


\section{8}

REVUE HYBRIDE DE L'ÉDUCATION

Ces cinq propositions caractérisent en 1984 les communautés de petite pêche bretonnes. La pêche est à la fois décrite par une transmission familiale "sans apprentissage » et par le souci technologique de certains pêcheurs-innovateurs. Ces propositions sont d'autant plus intéressantes à confronter à des récits de pêcheurs québécois au début du XXle que ceuXci ont expérimenté et expérimentent des restructurations économiques, des évolutions techniques relatives, en particulier, à des moratoires sur la pêche et au développement des techniques de chalutage (Merri et Képhaliacos, 2016).

\section{Méthodologie}

Huit pêcheurs d'une communauté québécoise se sont entretenus avec la chercheure pendant une heure et demie dans un local situé dans le port de pêche ou sur le chalutier. Ils pêchent actuellement la crevette sur un chalutier. II s'agit de six capitaines de pêche-propriétaires.

Ces capitaines de pêche ont été choisis selon trois caractéristiques : a) des précurseurs sur le plan technique (Laurent et Étienne); b) des interlocuteurs du pouvoir politique (Laurent et Alexandre) ; c) des capitaines ayant été hommes de pont pendant une grande partie de leur carrière (François, Jacques, Philippe). La variation de l'âge (Laurent, François, Jacques, Philippe, Alexandre et Étienne par âge décroissant) permet de distinguer ceux qui ont connu les techniques de pêche antérieures au chalutage de ceux qui n'ont pratiqué que ces dernières et sont diplômés de l'école de pêche.

Deux «hommes de pont » aspirant à le devenir : Benjamin, le plus jeune des deux, étant fils de propriétaire et Pierre ${ }^{2}$, s'apprêtant à devenir capitaine sur le bateau d'un propriétaire. Ces caractéristiques sont relatives tant à une possible imprégnation familiale dans l'enfance qu'à une variation dans la délégation des tâches par le capitaine.

Cet entretien comportait deux étapes : la description de sa trajectoire vers le statut de capitaine et la description de ses pratiques actuelles en lien avec ses instruments de travail (sondes, chalut, équipage, savoirs professionnels, etc.). La recherche a reçu une autorisation éthique de la Faculté de Sciences Humaines de I'UQAM et les participants ont donné leur consentement.

La première étape visait à obtenir le récit d'apprentissage du métier et à dégager les événements biographiques liés à des apprentissages et à des obstacles particuliers ; "Quand avez-vous commencé à pêcher ? Dans quelles situations avez-vous appris? Vous a-t-on expliqué ou montré ce qu'il fallait faire ? Et lorsque vous avez fait tout seul pour la première fois...? Avez-vous déjà modifié l'engin de pêche, "fait de la roue"... ? » La seconde

\footnotetext{
${ }^{2}$ Tous les prénoms ont été modifiés.
} 


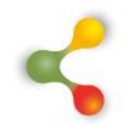

REVUE HYBRIDE DE L'ÉDUCATION

partie visait à contextualiser, dans la perspective de l'instruction au sosie (Oddonne et al., 1981), l'activité des pêcheurs lors d'un voyage de pêche à venir. Ainsi, le chercheur donnait au pêcheur la consigne suivante : "Imaginez que je sois votre sosie. Je prends votre place samedi prochain, mais les membres de votre équipage ne doivent pas se rendre compte de la substitution. Alors, que dois-je faire?" Les pêcheurs ont décrit les capacités qu'ils mobilisent au quotidien pour faire leur métier, mais aussi leur activité empêchée ou souhaitée (Clot, 2007). Spontanément, ils ont éclairé leur activité actuelle par leur activité passée et par leurs apprentissages, dans un esprit de comparaison et de complémentarité.

Les entretiens ont été intégralement transcrits puis segmentés selon les cinq propositions relevées dans les travaux de Delbos et Jorion (1990). Les segments ont fait l'objet d'une analyse thématique (Paillé et Mucchielli, 2015) dans NVivo puis ont été mis en vis-à-vis des propositions de Delbos et Jorion.

Les premiers résultats de la recherche ont été présentés et discutés dans le cadre d'un symposium réunissant professionnels et chercheurs. Plusieurs articles de l'ouvrage collectif issu de ce symposium sont rédigés ou co-rédigés par des membres de cette communauté de pêche (Merri et Képhaliacos, 2016).

\section{Résultats}

Dans cette section, nous présentons et commentons les résultats relatifs aux pêcheurs de la communauté québécoise en les plaçant en visà-vis des cinq propositions dégagées de l'analyse de Delbos et Jorion (1990).

\section{Chez les pêcheurs québécois, l'engagement dans le métier ne relève plus seulement d'une identification communautaire, mais aujourd'hui, nécessairement, d'une transition biographique pour soi.}

Tous les participants sont des enfants voire des petits-enfants de pêcheurs. Dès qu'ils entament le récit de leur parcours, six d'entre eux affirment leur identité communautaire (Dubar, 2010) à la suite des aînés et d'un monde disparu :

Pierre : Ben moi, cela a commencé au tout début... ben moi, mon grand-père maternel était pêcheur dans le temps, dans le temps qu'il y avait la morue avec les anciennes barges, les toutes petites barges d'avant.

Une identité biographique pour soi (Dubar, 2010) s'exprime également, conformément à l'évolution des formes d'identités dans le reste de la société. De nouvelles influences apparaissent : l'allongement de la scolarité, le souhait du père que le fils fasse des études ou qu'il ne fasse le 


\section{$<$}

REVUE HYBRIDE DE L'ÉDUCATION

métier que s'il lui plaît, les difficultés économiques de la filière, la réticence de la mère face au danger. Les premiers contacts avec la pêche consistent donc à faire des «tours » pour voir, à donner un «coup de main » pendant les vacances - la pêche étant alors associée au beau temps - et à monter à bord pour voir si on «pogne la twist ${ }^{3}$ » :

François, devenu capitaine, envisage aujourd'hui de faire "faire un tour» à son fils: Lui, il veut voir comment jeter les chaluts. Après ça, je vais lui montrer la timonerie, je vais lui expliquer les instruments et tout ça, comme je te dis, il va juste s'amuser et regarder à 11 ans, il a le temps encore puis de toutes façons, un peu plus tard, il décidera.

Jacques a tout de suite éprouvé un sentiment de liberté : Il (mon père) me dit : «viens essayer et si tu aimes ça, et lui voulait que j'aille à l'école et que je sois instruit. (...) Moi j'aimais ça, pour moi c'est un beau métier, le matin avec le soleil, c'est sûr qu'on connaît des tempêtes, mais par mer calme, je trouvais ça beau comme une liberté.

L'imprégnation sociale précoce est peu décrite par les pêcheurs québécois, par différence avec les récits recueillis par Delbos et Jorion (1990). Les pêcheurs insistent plutôt sur la nécessité personnelle d'engagement dans le métier. C'est d'abord à terre que le jeune homme a le désir d'embarquer, de voir et d'éprouver le métier ( «je cherchais à savoir ce que c'était», «il veut voir comment jeter les chaluts») et les aînés favorisent des situations pour qu'il fasse la part entre le plaisir et la souffrance ("c'est sûr qu'on connaît des tempêtes, mais par mer calme, je trouvais ça beau comme une liberté »).

\section{Le «non » du père devient ici un « oui si... » du capitaine.}

Les équipages des bateaux des participants comportent de trois à cinq membres, incluant le capitaine et les hommes de pont. Aucun équipage ne comporte plus d'un homme de pont membre de la famille du capitaine. II peut s'agir de son fils, ce dernier envisageant une transmission de l'entreprise, d'un frère ou d'un beau-frère. De plus, la main d'œuvre est aujourd'hui plus rare, car "ce qui a aidé au déclin des employés de la pêche, c'est la morosité de la pêche. » (Laurent).

Le capitaine et chacun des hommes de pont doivent chacun tenir leur place, le capitaine étant « posté » dans la cabine, les autres sur le pont.

\footnotetext{
${ }^{3}$ Pogner la twist : Maîtriser, apprendre les subtilités d'un processus ou d'un appareil et en obtenir un contrôle plus naturel et intuitif (https://www.wikebec.org/pogner-la-twist/definition/)
} 


\section{$<$}

REVUE HYBRIDE DE L'ÉDUCATION

La solidarité des membres de l'équipage décrite par Étienne est exemplaire du concept de solidarité organique de Durkheim (1893) :

Étienne, capitaine: Quand je fais de la navigation, je suis posté à l'avant parce que je regarde mes instruments électroniques. Pour les manipulations d'enrôleur, c'est ici que ça se passe. Donc toutes les manettes qui me permettent d'actionner les engins hydrauliques, les enrôleurs de chalut. Et les deux copains, matelots sur le pont sont postés chacun de leur côté pour surveiller ce qui se passe.

En effet, dans le cas de la pêche, les conditions périlleuses imposent une coopération. Sur le bateau, « on se parle, on s'entend »(Benjamin). La coopération de l'équipage est une attention partagée entre le pont et la cabine, médiatisée par le langage :

Étienne : II y a un langage personnel à chaque navire. Ici c'est très clair, "monte », "descends ", c'est beaucoup plus évident. On réserve les cris à quelque chose de grave. On ne crie jamais inutilement ici. Crier c'est pour que ça arrête tout de suite, il faut que ça change, il y a un petit danger imminent, un enjeu pour la santé ou pour la vie, ou pour un engin de pêche bloqué, quoi que ce soit.

L'évolution des places à bord correspond à la délégation de certaines de ses tâches par le capitaine. Le «non » du père (Delbos et Jorion, 1990) devient ici un « oui si... » du capitaine. Quatre catégories de «oui, si... » accordent alors conditionnellement à un homme de pont les fonctions de remplaçant, d'informateur, de collaborateur du capitaine ou d'aspirant-capitaine.

Le «oui si... » permet, en premier lieu, le repos du capitaine, à condition que celui-ci ne soit pas trop préoccupé et que le marin délégué soit compétent. Ainsi, le père de Benjamin ne se fait pas remplacer certaines nuits, car « (il) dort quatre heures par jour maximum. Quand il y a du poisson, (il dort) un peu, parce que s'il ne le prend pas, il ne dormira pas. Ça travaille dans les neurones pas mal. " Alexandre, quant à lui , choisit les hommes susceptibles de le remplacer pour des situations spécifiques, mais son corps reste vigilant dans son sommeil : "Tu te réveilles régulièrement aux cinq minutes (si c'est un jeune avec moins d'expérience) et comme je dis, s'il vire de bord, (...) il y a toutes sortes de petits détails qu'on a automatiquement. »

Le « oui si... » favorise, en second lieu, l'homme de pont possédant des connaissances techniques à la hauteur de celles du capitaine pour une coopération encore plus efficiente. En effet, tous les capitaines accueillent avec intérêt les jeunes formés à l'école de pêche ou ayant suivi des 


\section{$<$}

REVUE HYBRIDE DE L'ÉDUCATION

formations. Dans cet exemple, l'homme de pont devient l'œil du capitaine sur l'engin de pêche en contrebas de la cabine :

Alexandre : J'ai embarqué un jeune un moment donné qui était à l'école et qui connaissait le plan de son chalut parce qu'il avait étudié ça, il était capable d'essayer de deviner lui aussi de dire "ça, c'est le belly numéro $1^{4} »$ et il était bon. (...) Oui, parce que quand on roule (le chalut), il va me dire «bon, Alexandre, le belly numéro 1 est brisé ». Je continue à rouler, j'embarque le poisson, après on répare. Sinon, je roule et je ne sais pas où c'est brisé si personne ne me le dit.

Le « oui si... » concerne, en troisième lieu, les hommes de pont ayant un intérêt pour la nouveauté technologique. L'homme de pont fournit alors au capitaine un accompagnement expérimental et réflexif lors des transitions sur les engins de pêche, les deux professionnels étant tous deux novices:

Pierre - homme de pont : C'est du nouveau (ce type de chalut) pour lui (mon capitaine) (...), je vais beaucoup m'attarder à regarder ce qui va se passer, comment ils vont manœuvrer, comment ils vont agir avec les courants, avec le vent (...) j'ai hâte de voir...

Enfin, le «oui si...» concerne l'accompagnement des jeunes qui souhaitent devenir capitaines de pêche. Cette «ambition» oriente leur travail et leurs apprentissages :

Pierre: lorsque les gars peuvent se débrouiller seuls en bas, il (mon capitaine) me fait signe et il me dit : «viens en haut », moi je me déshabille, je monte dans la cabine et je prends les commandes puis je commence à manœuvrer tranquillement.

Cependant, comme l'observent également Delbos et Jorion (1990), les fils de capitaine rapportent des délégations de tâches moins progressives, le père créant une situation pour que le fils soit « prêt » même si celui-ci manque encore de confiance en lui. Les situations de travail sont transformées (Goffman, 2009) en épreuves de passage et le jeune pêcheur n'en découvre la « magie sociale » (Bourdieu, 1982) qu'a posteriori :

Étienne: La première fois que j'ai déplacé le bateau seul, j'avais, je crois 19 ans, 18-19 ans, c'était ce bateau-ci, mon père m'avait dit : «tiens, si tu te sens prêt, tu le sors du débarquement, tu vas le stationner là-bas ". Je regarde ça et je me dis qu'il me semble que

\footnotetext{
${ }^{4}$ Partie du chalut.
} 


\section{$\&$}

REVUE HYBRIDE DE L'ÉDUCATION

ça a l'air serré entre les deux bateaux, ça ne doit pas rentrer. "Oui, oui, ça va rentrer pas de problème ". Donc il dit, « prends ton temps, les gars. ils vont t'aider, ça prendra le temps que ça va, ok si t'as confiance. " J'ai déplacé le bateau, ça été très long, on a fait ça comme des bijoutiers, un mètre à la fois. Ça été très long. Quand mon père est revenu de commissions, je le vois encore lever sa casquette comme ça, tout surpris. II dit «ben, tabarouette, je ne pensais pas que ça rentrerait là ». J'ai tout compris. II voulait m'envoyer faire quelque chose qui ne se faisait pas pour que je sois obligé d'aller ailleurs, faire autre chose, trouver une solution. Mais on a réussi à le faire. Cette journéelà, il s'est passé quelque chose de très important.

Enfin, le changement des places sur le bateau n'implique pas toujours à terme un changement de statut, car certains pêcheurs choisissent de rester hommes de pont ou ne peuvent acheter un bateau. De plus, le statut de capitaine s'accompagne de la volonté et de la compétence critique à coordonner et à gérer les relations humaines au sein de l'équipage ( « J'essaie de jouer le psychologique des gars, la stratégie. », Alexandre), à trouver le poisson et à faire les bons choix technologiques. Certains hommes de pont souhaitant devenir capitaines prennent alors l'initiative de changer de place sur le pont. En effet, le capitaine dispose d'une vue d'ensemble de l'instrument de travail et il ne faut donc pas se surspécialiser.

\section{Les modalités d'apprentissage varient selon des catégories de situations professionnelles.}

Les pêcheurs décrivent les catégories de situations composant leur champ professionnel. Ils associent à ces catégories des modalités d'apprentissage à la fois variées et spécifiques (tableau 2). Les deux premières catégories appartiennent aux hommes de pont: elles concernent les manœuvres du chalut et le conditionnement du poisson (catégorie 1) et la réparation du chalut (catégorie 2). Les bris graves de matériel (catégorie 3) engagent pleinement le capitaine, car ces réparations conditionnent la possibilité de pêcher. La navigation (catégorie 4) et la stabilité du bateau (catégorie 5), la recherche des lieux de pêche (catégorie 6) et le choix puis l'utilisation de la technologie de pêche (catégorie 7) appartiennent, quant à elles, spécifiquement au capitaine.

Le «non-apprentissage du métier» et «l'apprentissage comme mémoire des erreurs réprouvées par le père », catégories repérées par Delbos et Jorion (1990) (tableau 1), sont absentes des témoignages des pêcheurs québécois. De plus, les formes d'apprentissage relatives aux catégories spécifiques aux hommes de pont accordent, contrairement à Delbos et Jorion (1990), une place importante à l'explication par les pêcheurs aguerris et à des formes scolaires d'apprentissage. 


\section{$<$}

REVUE HYBRIDE DE L'ÉDUCATION

En effet, les hommes de pont entrent dans le métier par une observation des procédures c'est-à-dire des suites d'actions régulières pour accomplir la tâche $\left(\mathrm{M} 1^{5}\right.$, tableau 2) puis le jeune pêcheur peut demander à l'accomplir, bénéficiant des explications de ses collègues en accompagnement de son geste :

Pierre: Moi, je tenais le fil, donc je les regardais faire. Par la suite, moi, par l'ambition personnelle naturelle, j'ai simplement dit : «je peux-tu l'essayer? » Donc, j'ai pris l'aiguille et je l'ai essayé moi-même, afin que... c'est eux qui me disaient : "tu devrais prendre là, tu devrais prendre là, tu devrais faire ça », par le cheminement qu'ils m'ont expliqué, cela m'a donné une petite idée.

La formation à l'école de pêche (M3) est valorisée, en particulier dans les situations relatives à la réparation des engins de pêche, car elle dote les pêcheurs d'une conceptualisation des composantes du chalut et permet d'effectuer les réparations et réglages selon les normes techniques. L'école appuie ainsi leur intérêt pour l'instrument de pêche et ses innovations technologiques. L'école offre également au novice du temps pour faire et refaire, essayer et réessayer. Certains pêcheurs aguerris mettent également en place un enseignement par explication en faveur du jeune pêcheur :

Alexandre : Quand j'avais de la misère, je disais : «là je ne suis plus capable ", là il m'expliquait : «non là, t'as mal fait ça, va là » et il m'expliquait tout comme il faut. Fait que quand je suis arrivé à l'école des pêches, j'avais déjà une base. Je savais coudre, là on m'a appris à coudre comme il faut.

Philippe : C'était un gars qui était patient (...) il est venu à côté de moi, il a dit: "prends une aiguille et commence tranquillement et si cela ne marche pas, viens me voir, j'attends ».

Les situations problèmes (catégorie 3 ) dont ils sont témoins à bord apprennent aux pêcheurs l'importance du temps de la réflexion (M4) :

Pierre décrit un bris grave du chalut: Le capitaine a dit : « Bon! Là, on va prendre le temps, on s'assied, on va réfléchir à notre problème » (...). Cette chose-là m'a appris que (...) prendre le temps, c'est toujours la clé du succès en mer, sur des bateaux de pêche.

\footnotetext{
${ }^{5}$ Les modalités d'apprentissages codées M1, M2... renvoient au tableau 2.
} 


\section{8}

REVUE HYBRIDE DE L'ÉDUCATION

Si les règles de navigation sont enseignées dans la formation professionnelle (catégorie 4), la stabilité du bateau (catégorie 5) est en partie, comme nous l'avons vu plus haut, inscrite dans le corps du pêcheur et acquise par frayage (M5). L'une des catégories discriminantes des capitaines apparaît être la capacité à trouver le poisson (catégorie 6), également décrite par Delbos et Jorion (1990) (tableau 1, ligne 4). Cette confrontation à la nature par des savoirs locaux (M6) correspond à une prise en compte globale et articulée de l'environnement naturel local et le repérage de régularités entre indices naturels et présence du poisson. Certains pêcheurs en seraient dotés et d'autres moins, selon les participants. Ces savoirs locaux sont acquis sans transmission explicite, ce qui fait dire qu'à l'école de pêche, "on n'apprend pas à pêcher». Les novices cherchent alors à « guesser ${ }^{6}$ le capitaine :

Laurent : Je posais énormément de questions mais lui (le capitaine), il ne disait rien. Juste un exemple, c'était quelqu'un qui n'aimait pas ça pêcher quand il y avait trop de bateaux. Et moi je me suis aperçu de ça, il ne m'a jamais dit ça de sa vie, jamais, jamais, jamais. On pouvait pêcher à une place et il arrivait des bateaux. II ne parlait pas un mot, pas un mot, pas un mot. Quand la noirceur prenait, on levait le chalut, on ramassait et il disait : " on s'en va là ». On arrivait là, il y avait du poisson en masse, on était tous seuls. (...) Là un moment donné, j'avais dit : «pourquoi il dit «il y en a là » et il y en a là ?

La catégorie 7 (choix, ajustement et utilisation de la technologie de pêche) fait l'objet de la section suivante.

Tableau 2 : Les modalités d'apprentissage déclarées par les pêcheurs québécois

\begin{tabular}{|l|l|l|l|l|l|l|l|}
\hline & $\begin{array}{l}\text { 1- } \\
\text { Mancu } \\
\text { vrer le } \\
\text { chalut, } \\
\text { conditi } \\
\text { onner } \\
\text { le } \\
\text { poisso } \\
\text { nép }\end{array}$ & $\begin{array}{l}\text { Ré } \\
\text { arer } \\
\text { le } \\
\text { filet }\end{array}$ & $\begin{array}{l}\text { 3- } \\
\text { Rép } \\
\text { arer } \\
\text { les } \\
\text { bris } \\
\text { grav } \\
\text { es } \\
\text { de } \\
\text { mat } \\
\text { ériel }\end{array}$ & $\begin{array}{l}\text { Navi } \\
\text { guer }\end{array}$ & $\begin{array}{l}\text { 5- } \\
\text { Resse } \\
\text { ntir le } \\
\text { mouve } \\
\text { ment } \\
\text { du } \\
\text { bateau }\end{array}$ & $\begin{array}{l}\text { 6- } \\
\text { Trou } \\
\text { ver } \\
\text { le } \\
\text { pois } \\
\text { son } \\
\text { et le } \\
\text { pêc } \\
\text { her }\end{array}$ & $\begin{array}{l}\text { Choisir } \\
\text { ajuster } \\
\text { et } \\
\text { utiliser } \\
\text { des } \\
\text { technol } \\
\text { ogies } \\
\text { de } \\
\text { pêche }\end{array}$ \\
\hline $\begin{array}{l}\text { M1 } \\
\text { Observati } \\
\text { on des } \\
\text { autres }\end{array}$ & $\mathrm{X}$ & & & & & & \\
\hline
\end{tabular}

${ }^{6}$ Guesser : deviner 
REVUE HYBRIDE DE L'ÉDUCATION

\begin{tabular}{|c|c|c|c|c|c|c|c|}
\hline $\begin{array}{l}\text { pour } \\
\text { repérer } \\
\text { une } \\
\text { procédur } \\
\text { e }\end{array}$ & & & & & & & \\
\hline $\begin{array}{l}\text { M2 } \\
\text { Observati } \\
\text { on active } \\
\text { des } \\
\text { autres } \\
\text { puis } \\
\text { démonstr } \\
\text { ation }\end{array}$ & $X$ & $X$ & & & & & \\
\hline $\begin{array}{l}\text { M3 École } \\
\text { pour un } \\
\text { apprentis } \\
\text { sage de } \\
\text { normes } \\
\text { Exercices } \\
\text { organisés } \\
\text { par un } \\
\text { autre } \\
\text { pêcheur }\end{array}$ & & $\mathrm{X}$ & & $X$ & & & \\
\hline $\begin{array}{l}\text { M4 La } \\
\text { réflexion } \\
\text { avant } \\
\text { l'action }\end{array}$ & & & $X$ & & & & $\mathrm{X}$ \\
\hline $\begin{array}{l}\text { M5 } \\
\text { L'apprenti } \\
\text { ssage par } \\
\text { frayage }\end{array}$ & & & & & $X$ & & \\
\hline $\begin{array}{l}\text { M6 Les } \\
\text { savoirs } \\
\text { locaux : } \\
\text { réactualis } \\
\text { ation de } \\
\text { la } \\
\text { confrontat } \\
\text { ion } \\
\text { personnel } \\
\text { le et } \\
\text { privée } \\
\text { avec la } \\
\text { nature }\end{array}$ & & & & & & $\mathrm{X}$ & $x$ \\
\hline $\begin{array}{l}\text { M7 } \\
\text { Consultati } \\
\text { on } \\
\text { d'experts } \\
\text { et }\end{array}$ & & & & & & & $x$ \\
\hline
\end{tabular}

Numéro 5 
REVUE HYBRIDE DE L’ÉDUCATION

\begin{tabular}{|l|l|l|l|l|l|l|l|}
\hline expérime & & & & & & & \\
ntations & en & & & & & & \\
situation & & & & & & & \\
locale & & & & & & & \\
\hline
\end{tabular}

Les pêcheurs québécois mobilisent à la fois une imputabilité, un pouvoir d'agir et un amour-propre à l'instar des pêcheurs de Delbos et Jorion.

Tous les pêcheurs rencontrés sont actuellement capitaines ou projettent de le devenir. Sept d'entre eux affirment ainsi devoir le produit de la pêche à leur propre action et se sentir responsables des hommes. Les critères moraux de leur imputabilité (Ricœur, 2005) sont conflictuels entre la performance et la sécurité, la productivité et le bien-être des hommes de pont, la quantité et la qualité du poisson. Dans ce dernier cas, l'imputabilité du pêcheur engage la durabilité de la pêche :

Étienne : Définitivement, il faudrait arrêter de viser les quantités, (...) il faut que ce soit plus axé sur la conservation de la ressource (...) Parce que je cible la plus grosse crevette. Parfois, ça fait en sorte qu'on amène des plus petites quantités, quand mes voisins rentrent des cales pleines, moi je ne me promène pas avec la cale pleine.

Ces critères moraux sont mis en œuvre grâce à un pouvoir faire, objet de l'« amour propre » du pêcheur. Cet amour-propre est longuement évoqué par les participants québécois. II s'exprime d'abord, comme dans les récits de Delbos et Jorion (1990) à l'égard de leur famille, par la fierté et la reconnaissance réciproques du père et du fils et par leur souhait de transmission de la mémoire du bateau. Ainsi, l'un des pêcheurs rédige un journal quotidien :

Jacques: Chaque voyage est rentré dedans, le nombre d'heures du moteur, les changements d'huile, comme là, Pascal était en vacances la semaine passée (...) moi, j'ai des enfants, ils aiment ça, je vais parler de pêche, j'ai écrit telle chose et ils sont contents de ça, que j'écrive ça, et cela les met au courant des affaires.

II s'exprime, en second lieu, envers leur communauté de pêche à travers la fierté de leur équipage d'appartenir à un bateau performant c'est-à-dire plus performant que les autres - et par leur souci d'être réactif technologiquement. 


\section{$\&$}

REVUE HYBRIDE DE L'ÉDUCATION

Leur amour-propre s'exprime enfin à l'égard des autres acteurs de la pêche, en particulier les législateurs et les scientifiques qui disent aux pêcheurs "comment pêcher», menaçant leur compétence et leur autonomie. En particulier, Laurent s'appuie explicitement sur le concept anthropologique de "savoirs locaux » (Barthélémy, 2005) et milite pour la reconnaissance de ces savoirs par les politiques et par les scientifiques.

On le voit, l'amour-propre des pêcheurs québécois a des conséquences sociales, écologiques, économiques et politiques. En effet, il les conduit à privilégier certaines pratiques de pêche, à les diffuser, à user de distinction dans la communauté des pêcheurs et, éventuellement, à engager un débat avec d'autres acteurs influents du secteur de la pêche.

\section{Les choix et les innovations technologiques sont source d'identités subjective et sociale et font évoluer le modèle opératif du pêcheur.}

Comme le prévoient Delbos et Jorion (1990), quelques capitaines investissent tout particulièrement les thèmes technologiques au cours de l'entretien. Le choix et la conception du chalut correspondent pour eux à une étape de leur construction identitaire de capitaine. Ainsi, Laurent a décidé de ne plus être "confiné » au golfe du Saint-Laurent et a besoin d'« équipements convenables" pour la région Atlantique. Étienne, capitaine également audacieux, privilégie les fonds accidentés :

Étienne: J'étais rendu à une étape où je voulais un chalut qui était plus solide sur l'effort accidenté, je savais que c'était celui-là. Un chalut qui n'était pas trop gros pour être quand même facile à traîner pour sauver du carburant, celui-là. Un chalut qui me donnait une belle hauteur pour être performant la nuit, celui-là. (...) C'est mes propres critères personnels qui m'ont amené vers lui.

Le choix du chalut est également, pour les pêcheurs qui mandatent (Lorino, 2013 ; Merri et al., 2015) des techniciens de pêche, une occasion d'explicitation de leurs critères selon les paramètres d'un logiciel d'aide à la prise de décision technique. Lorsque le chalut est construit, celui-ci reste un objet de préoccupation pour le capitaine, car l'engin de pêche nécessite un ajustement de performance, selon des paramètres conscients. Ces ajustements requièrent, selon tous les capitaines, un apprentissage tout au long de la vie. Alexandre fait appel à l'expertise de ses frères (M7) :

Alexandre : Encore aujourd'hui, quand j'ai un problème et que j'ai de la misère à le régler, je discute avec mes frères, j'en appelle un ou l'autre et je leur explique le problème et, tout en jasant, des fois ça prend du temps, on jase, on jase et «ah! On a oublié ça. Check ça, ça doit être ça. » Des fois tu sais, c'est un détail et ce n'est 


\section{8}

REVUE HYBRIDE DE L'ÉDUCATION

pas en disant ton problème à quelqu'un qu'il va te le trouver, mais en parlant avec, tout en parlant des fois, il y a une phrase qu'un des deux va dire et «Ah! C'est vrai, on n'a pas checké ça. » Ou des fois, il va te le trouver tout de suite.

Certains pêcheurs sont des "pionniers " technologiques dans la communauté et s'équipent de nouveaux engins, n'hésitant pas à se rendre en Europe pour observer le comportement de ceux-ci dans des centres de recherche. La profondeur, la hauteur et l'ouverture du chalut, le vent, les courants, la température sont autant de paramètres qu'ils n'utilisent plus seulement pour concevoir leur chalut, mais aussi pour l'ajuster en temps réel, au cours des traits de chalut ${ }^{7}$. Ainsi, les pêcheurs qui appuient l'importance des savoirs locaux dans le succès de la pêche insistent également sur l'apport des moniteurs, car « il faut tout le temps que tu sois à l'affût, c'est pour ça que c'est important de vișualiser et avoir ces données-là en temps réel. » (Laurent). Si Laurent et Étienne, en particulier, évoquent leur scepticisme initial et leur difficulté face aux mesures qui semblent parfois contredire leur perception, ils ont appris à faire " confiance » aux nouveaux dispositifs. Les paramètres de ces nouveaux équipements doivent donc s'articuler avec leurs savoirs locaux. François, quant à lui, insiste sur la fonction de mise en mémoire des traits de chalut passés:

François : Tous les systèmes qu'on a aujourd'hui, je te dirais, tous les ordinateurs, on est assez bien équipés en électronique, c'est super (...) tout est rentré en mémoire, il y a toutes de belles lignes, de tracés qu'on appelle. (...).

Ces pêcheurs témoignent de leur nouvelle compréhension de leurs difficultés passées à pêcher, ce qui les conduit à différencier "trouver le poisson » et «pêcher le poisson », car le prédateur peut trouver le poisson sans savoir le capturer.

\section{Discussion}

Plus de trente ans après, les propositions de l'ouvrage de Delbos et Jorion (1984) ont été mises en vis-à-vis des discours de huit pêcheurs d'une même communauté de pêche québécoise.

Les pêcheurs ne s'inscrivent plus seulement dans une identité communautaire, mais également dans une identité biographique pour soi (Dubar, 2010), à l'instar des orientations professionnelles dans la société. En particulier, ils commencent par «faire des tours » et ces moments de mise à l'épreuve du « moi comme pêcheur » ont une résonance symbolique

\footnotetext{
${ }^{7}$ Période effective de pêche pendant laquelle le navire traîne le chalut.
} 


\section{8}

REVUE HYBRIDE DE L'ÉDUCATION

(Méloni et Rassial, 2014) : le pêcheur s'individualise en ressentant, dans l'émotion et dans le geste, la possibilité, à terme, de faire ce métier.

Les équipages de bateaux de cette communauté ne sont plus uniquement familiaux, la transmission de l'entreprise de pêche se faisant encore, néanmoins, souvent du père vers le fils pour des raisons en partie capitalistiques. Les pêcheurs décrivent une solidarité organique (Durkheim, 1893) entre hommes de pont et capitaine. Des habitudes de communication se créent entre ces hommes porteurs de fonctions différentes, car " s'il (le professionnel) ne sait pas où tendent ces opérations qu'on réclame de lui, s'il ne les rattache à aucun but, il ne peut plus s'en acquitter que par routine. " (Durkheim, 1893, p.117, livre III). Tous les participants favorisent donc une variation des places dans laquelle, contrairement aux récits d'apprentissage recueillis par Delbos et Jorion, la formation scolaire des hommes de pont à l'école de pêche et par l'obtention de brevets maritimes est appréciée. Au-delà de l'observation des autres membres de l'équipage, des formes explicites d'apprentissage sont également décrites.

Dans le même temps, les pêcheurs québécois revendiquent, à l'instar des témoignages de Delbos et Jorion (1984), une pratique en partie subjective de la pêche par l'acquisition de savoirs locaux - qui appartiennent en propre au capitaine et en fondent la distinction (Bourdieu, 1979 ; Mauroy, 2014) tant sur le bateau que dans la communauté. En effet, cette communauté, à travers certains de ses membres précurseurs (Merri et al., 2016), s'est elle-même mobilisée pour légitimer ses savoirs locaux en s'appuyant, en particulier, sur la communauté de recherche en anthropologie maritime (Levain et Laval, 2018).

Enfin, l'investissement technologique, fortement privilégié dans cette communauté de pêche, est une nouvelle occasion d'activité constructive (Pastré, 2012) pour les pêcheurs, car ils développent alors un rapport d'objet à leur chalut et non un simple rapport instrumental (Rabardel, 2005). En effet, ils mettent en œuvre à fois des savoirs locaux et de nouveaux paramètres de description de l'environnement et du comportement de l'engin de pêche pour leur intégration à un modèle opératif (Pastré, 2012) orienté vers deux buts: trouver et pêcher du poisson. Des travaux ultérieurs devraient donc se centrer sur cette intégration en composant, néanmoins, avec la nature secrète de la recherche du poisson pour les pêcheurs.

\section{Remerciements}

Nous remercions les professionnels de pêche rencontrés pour leur disponibilité au cœur de la saison de pêche et leur plaisir de raconter leur expérience. Nous remercions également le directeur de leur association pour son aide dans la prise de contact avec les professionnels et pour son accueil dans les locaux. 


\section{REVUE HYBRIDE DE L'ÉDUCATION}

\section{Références}

Archambault, J. (1997). Savoir local des pêcheurs de homard des Îles-de-laMadeleine: Vers une meilleure compréhension de l'état des stocks [Mémoire de maîtrise inédit]. Université Laval.

Barthélémy, C. (2005). Les savoirs locaux: Entre connaissance et reconnaissance. Vertigo, 6 (1), 59-64.

Biget, D. (2005). Entre l'école et la mer. Techniques et culture, 45.

Biget, D. (2009). L'enseignement professionnel des pêches maritimes en France (1895-2007). Essai d'anthropologie historique. L'Harmattan.

Boudreau, M. et Myre, G. (1998). Le ramendage. La réparation des filets de pêche. Cégep de la Gaspésie et des Îles.

Bourdieu, P. (1979). La distinction : Critique sociale du jugement. Les Éditions de Minuit.

Bourdieu, P. (1982). Les rites comme actes d'institution. Actes de la recherche en sciences sociales, 43, 58-63. https://doi.org/10.3406/arss.1982.2159

Bruner, J. S. (1996). Meyerson aujourd'hui: Quelques réflexions sur la psychologie culturelle. Dans F. Parot (dir.), Pour une psychologie historique. Ecrits en hommage à Ignace Meyerson (p. 193-207). Presses Universitaires de France.

Clot, Y. (2007). De l'analyse des pratiques au développement des métiers. Éducation et didactique, 1(1), 83-94.

Del Corso, J.-P. (2008). Éducation à l'ordre institutionnel et types de discours en économie et en gestion. Comment l'enseignant prépare-t-il des conseillers en agriculture aux situations d'incertitude? [Thèse de doctorat inédite]. Université Toulouse le Mirail.

Delbos, G. et Jorion, P. (1984). La transmission des savoirs (Première édition). Maison des Sciences de l'Homme.

Delbos, G. et Jorion, P. (1990). La transmission des savoirs (Seconde édition). Éditions de la Maison des sciences de l'homme.

Deschamps, G. (2009). Les filets maillants. Éditions Quæ.

Dubar, C. (2010). La crise des identités ( $4^{\mathrm{e}}$ éd.). Presses Universitaires de France.

Duhamel du Monceau, H.-L. (1776). Traité des pêches et histoire des poissons (sections I, II et III)- Tome V de la Description des Arts et Métiers. J.-E. Bertrand.

Durkheim, É. (1893). De la division du travail social. Presses universitaires de France. 


\section{REVUE HYBRIDE DE L’ÉDUCATION}

Geertz, C. (1986). Savoir local, savoir global. Presses Universitaires de France.

Goffman, E. (2009). Les cadres de l'expérience (I. Joseph, Trad.). de Minuit.

Lave, J. et Wenger, E. (1991). Situated Learning: Legitimate Peripheral Participation. Cambridge University Press.

Levain, A. et Laval, P. (2018). Jusqu'où va la mer ? Une exploration des marges de l'anthropologie maritime. Revue d'ethnoécologie, 13. https://doi.org/10.4000/ethnoecologie.3449

Lorino, P. (2013, avril). Transaction and transformation: A pragmatist perspective on learning as transforming action and transacting forms. International Conference on Organizational Learning, Knowledge and Capabilities, Washington.

Mauroy, H. (2014). L'amour-propre : Une analyse théorique et historique. Revue européenne des sciences sociales, 52-2, 73-104. https://doi.org/10.4000/ress.2800

Mayen, P. (2016). Agriculture plus agroécologique et transformation des exigences cognitives. Dans M. Merri et C. Képhaliacos (dir.), Nouvelles raisons d'agir des acteurs de la pêche et de l'agriculture. Quæ.

Méloni, D.et Rassial, J.-J. (2014). Le lien social et le désir dans la recherche d'une orientation professionnelle. Travailler, 32(2), 131. https://doi.org/10.3917/trav.032.0131

Merri, M. et Doualot, A. (2013). Les ambitions documentaires et didactiques de la description du métier de pêcheur dans le Traité des pêches. Éducation et didactique, 7(3), 31-54.

Merri, M. et Képhaliacos, C. (Éds.). (2016). Nouvelles raisons d'agir des acteurs de la pêche et de l'agriculture. Quæ.

Merri, M., Veillette, S. et Cotton, R. (2016). Une histoire à faire vivre : Hériter du métier de pêcheur au Québec et le cultiver (M. Merri et C. Képhaliacos, éds.). Quæ.

Merri, M., Veillette, S. et Laurendon-Marques, C. (2015). La maintenance des chaluts comme activité collective dans un port méditerranéen. Revue internationale d'ethnographie (RIE), 5, 138-155.

Oddonne, Y., Re, A. et Briante, G. (1981). Redéfinir l'expérience ouvrière : Vers une autre psychologie du travail. Éditions Sociales.

Paillé, P. et Mucchielli, A. (2015). L'analyse qualitative en sciences humaines et sociales. Armand Colin.

Pastré, P. (2012, juin). Apprentissage et développement chez les adultes au travail et en formation. $2 \mathrm{e}$ colloque international de didactique professionnelle, Nantes. http://didactiqueprofessionnelle.ning.com/page/colloque-2012-nantes

Rabardel, P. (2005). Instrument subjectif et développement du pouvoir d'agir. Dans P. Rabardel et P. Pastré (dir.), Modèles du sujet pour la conception : 


\section{REVUE HYBRIDE DE L’ÉDUCATION}

Dialectiques, activités, développement (p. 11-29). Octarès.

Ricœur, P. (2005). Devenir capable, être reconnu. Esprit, 7, 125-130.

Rostaing, C. (2012). Carrière. Sociologie, Les 100 mots de la sociologie. https://sociologie.revues.org/1197

Ulmann, A.-L. (2009). Les savoirs de la pratique professionnelle. Dans G. Brougère et A.-L. Ulmann, Apprendre de la vie quotidienne (p. 155). Presses Universitaires de France.

Wenger, E. (2005). La théorie des communautés de pratique. Presses de l'Université Laval. 